\title{
SPECTRAL CLASSIFICATION IN THE SMALL MAGELLANIC CLOUD
}

\author{
P. DUBOIS, M. JASCHEK, and C. JASCHEK
}

Observatoire de Strasbourg, France

\begin{abstract}
This paper presents results obtained in classifying 26 supergiants of the Small Magellanic Cloud. The main results are:

- The hydrogen lines are not as sharp as expected for such luminous stars.

- Generally, for the A-type stars, the calcium $\mathrm{K}$-line is too strong compared to the other metallic lines.

- There are differences in the behaviour of the different metals.

- Often, the stars present variations in their spectra.

- 50\% of the stars brighter than $m_{v}=11.3$ have an enhancement of the H8 Balmer line, which is one of the characteristics of a shell star.

- The comparison with the Radcliffe classification and Dachs classification is, except in very few cases, in good agreement.
\end{abstract}

It is well known that there are problems connected with the chemical composition of the SMC stars, the opinions ranging from solar composition to various degrees of underabundance of metals. Because chemical composition analyses require high dispersion and these in turn very long exposure times, we have adopted a simpler method. We have tried to compare a sufficiently large number of SMC supergiants to galactic supergiants. This paper gives some results concerning $26 \mathrm{~B}$ and A-type SMC supergiants.

The objects were selected from the list of Florsch (1972), who gives the radial velocity. measured with Fehrenbach's objective prism, and from the list of Sanduleak (1968). Most of the stars are common to both lists. The sample of galactic supergiants is composed of 38 stars classified by Morgan.

The spectra were obtained at the ESO Observatory in Chile with a slit grating spectrograph at the Cassegrain focus (RV Cass) which gives a dispersion of $74 \AA \mathrm{mm}^{-1}$. The projected slit width on the film is $16 \mu(=1.2 \AA)$. Because of the exposure times, the spectra have to be trailed in height only over $200 \mu$. The spectra show the whole region from $\lambda$ 3600-4900 $\AA$. Some spectra taken by Azzopardi with the same instrument were kindly lent by him for this work.

The spatial distribution of the SMC stars is the following: 3 are located in the wing, 20 in the bar, mainly at the two ends, and 3 lie outside. Among them we have 8 B-type stars and 18 A-type. The proportionally larger number of A-type stars comes from the fact that we chose them from the list of Florsch. For the A-type stars the Balmer line have the largest contrast and are thus more easy to measure by the objective prism technique.

When classifying these stars one runs immediately into problems, because the stars do definitely not show spectra like those of their galactic counterparts.

The first characteristic is that the hydrogen lines are not so sharp as could be expected of such high luminosity stars, except in a few cases. One finds stars having an absolute 
magnitude of about -7 which have hydrogen lines broader than those of galactic supergiants of luminosity class $\mathrm{lb}$, to which we assign generally an absolute visual magnitude of about -5 .

As a result of this, the number of the last visible Balmer line is not larger than in galactic bright supergiants of similar spectral type. Furthermore, the Balmer jump in many cases corresponds to an earlier spectral type.

The line broadening described is not restricted to the hydrogen lines, but is shared in general by all the other lines.

A second striking characteristic is, in the A-stars, the absence or extreme weakness of the metallic lines. A closer analysis reveals however that this weakening is not uniform for all metals. In particular one finds in the A-stars, if one designs by $S(X)$ the spectral type obtained from the lines of element $X$, that:

$$
S(\mathrm{Ca}) \stackrel{\sim}{\sim} S(\mathrm{Ti} \text { II })>S(\mathrm{Fe} \text { II) }
$$

Not only is Fe II weaker than expected, but, moreover, $\mathrm{Fe}$ I lines are almost never visible.

Si II and Mg II lines are often - but not consistently - weaker than Ti II. The difference between $S$ (Ti II) ar:i $S$ (Fe II) is of the order of 2 tenths of spectral type, a fact which is easily visible in the interval A0-A2.

In the B stars, the weakening of elements other than hydrogen and helium is harder to detect. However, one does not see in the stars taken any enhancement of $\mathrm{C}, \mathrm{N}$ or $\mathrm{O}$ as could be expected in view of the luminosity of these stars. It should be added that perhaps also the $\mathrm{Si}$ is weaker than expected. For two of them Osmer (1973) found weak lines of $\mathrm{N}, \mathrm{O}, \mathrm{Si}$ and probably $\mathrm{Mg}$ and $\mathrm{C}$.

The third important point is that the Ca II lines are present not only in the A-type stars, but also in the B-type stars, except in two stars. But in both of our two exceptions, Feast et al. (1960) found on their spectra a K-line. The radial velocity of the K-line for all the A- and B-type stars coincides with the one determined from the other lines, within the errors of measurements.

A fourth characteristic is the spectrum variability. Since we have up to five spectra for each star, this becomes easily observable. The variations occur over time scales of one or several days. This kind of activity of the supergiants is well known from the studies of Rosendahl (1973), Hutching $(1971,1973)$ and others. Looking for a common characteristic of the stars which do vary, one finds that 5 out of 7 stars, earlier than B5, show at least on one plate an enhancement of the $\mathrm{H} 8$ line, which is a well-known characteristic of shell stars. However, not all other characteristics of shell stars - sharpness of lines, underlying broad lines, presence of numerous metallic lines - are always present. The enhancement of $\mathrm{H} 8$ occurs even in two A-type stars, so that if we consider all stars brighter than $11^{m} \cdot 2$, which corresponds to about $M_{\nu} \approx-8.3$ if one puts all cloud stars at the same distance, one finds that $50 \%$ of the stars do show this characteristic.

The existence of spectrum variability explains at least part of the differences found when comparing the present classification with those of Feast et al. (1960) and with 
Dachs (1970). There exists a systematic effect with the Radcliffe classification in the sense that our classifications are about one tenth later; the remaining dispersion is 1.5 tenth. The systematic effect is probably due to the fact that this classification relies more upon the intensity of the K-line than does that of Feast et al. (1960). The spectral types given by Dachs are based upon photometric measurements which depend mainly upon the Balmer discontinuity. Their agreement with the present work is poorer; the dispersion is 2.5 tenth, due problably to the fact that one compares classifications based upon different criteria.

Summarizing, it can be said that we have a certain number of facts which can only be interpreted when good atmospheric models of very bright supergiants become available. Since these do not yet exist, one can only conclude that one does not know if the anomalies found are due to a deficiency in metals or an abnormal atmospheric structure which enhances certain metals preferentially over others.

\section{References}

Dachs, I.: 1970, Astron. Astrophys. 9, 95.

Feast, M. N., Thackeray, A. D., and Wesselink, A. J.: 1960, Monthly Notices Roy. Astron. Soc. 121, 337.

Florsch, A.: 1972, Publication de l'Observatoire Astronomique de Strasbourg, Vol. 2 fasc. 1.

Hutching, J. B.: 1971, in M. Hack (cd.), Colloquium on Supergiant Stars, Osservatorio Astronomico di Trieste, Trieste, p. 38.

Hutching, J. B. and Laskarides, P. G.: 1973, Publ. Dominion Astrophys. Obs. 14, 107.

Osmer, P. S.: 1973, Astrophys. J. 184, 127.

Rosendhal. J. D.: 1973, Astrophys. J. 186, 909.

Sandulcak, N.: 1968, Astron. J. 73, 246.

\section{DISCUSSION}

Przybylski: You find that the $\mathrm{K}$-line of $\mathrm{Ca} \mathrm{Il}$ is too strong. I think one can generalize this statement by saying that lines arising from the ground level have the tendency to be too strong. This may be the reason why in the investigation of the brightest LMC supergiant, HD 33579, by Dr B. Wolf and me, we found an overabundance for aluminium.

Bidelman: I believe it is possible that the abnormally strong $\mathrm{K}$-line that you find is simply due to interstellar calcium, as it is known that the small Magellanic Cloud has a large amount of gas.

Dubois: I can only comment that in view of what I said it looks doubtful that this is the real explanation.

Cayrel: Could you tell me your personal opinion about a possible metal/hydrogen deficiency in the SMC?

Dubois: Probably the best answer is contained in the last paragraph of my talk.

Cayrel: Who found that the SMC is metal poor?

Przybylski: I found that the brightest star, HD 7583, is metal poor by a factor of 10 . However, Dr B. Wolf found normal abundances in this star. The discrepancy in the results is (at least) partly due to the difference in fixing the level of the continuum. My continuum cuts the photographic noise so that half of it is above and half below the adopted continuum. To the best of my knowledge Dr Wolf puts the continuum higher. 
Bell: Parsons and I believe the SMC cepheids may be metal deficient by a factor of $\sim 4$ in view of their blue colour relative to galactic cepheids.

Bell: (to Przybylski): Why don't you use your calibration spectra to estimate grain noise instead of stellar spectra in which weak lines may be present?

Przybylski: I did some investigations of that kind. I concluded that in the investigation of narrow spectra (of, say, $0.3 \mathrm{~mm}$ width) the photographic noise can introduce spurious lines of the order of about $7 \mathrm{~m} \AA$ if I remember well, provided that we fix the continuum in the way described in my answer to Mrs Cayrel's question. However, the strength of the spurious lines may be five times larger if we draw the continuum through the highest peaks. In the investigation of stellar spectra we can expect similar errors.

The photographic noice is of no importance if we investigate broad spectra. Unfortunately in spectroscopic investigations there is no substitute for good spectra.

Walborn: (1) The lines could appear broader if the densities of the SMC spectra were systematically lower than those of the bright standards.

(2) I would be cautious about interpreting as real, variations of weak features on narrow, lowdispersion spectrograms.

(3) I have obtained $78 \AA \mathrm{mm}^{-1} 1.2 \mathrm{~mm}$ wide spectrograms of two B0 supergiants in the SMC; the Si IV lines have strengths similar to those in galactic standards of luminosity classes IV and $V$, and the carbon and nitrogen features are very weak. These results are in agreement with those of Dr P. S. Osmer on metal deficiency in SMC supergiants.

Dubois: With the first point I agrec, and for this I have taken many spectra of each comparison star with various densities to eliminate this, utect.

To the second point, some variations are very obvious, so that the reality cannot be questioned.

The third point is in agreement with what we have observed.

McCarthy: Can you explain fully how your estimate of the Balmer jump influenced your spectral classification of these stars? You mentioned that the Balmer jump corresponds to an earlier spectral type for several SMC stars.

Dubois: For 'A' stars the Ca $\mathrm{K}$ line is the chief criterion adjusted later by reference to Balmer jump.

Wolf: (1) A possible reason for some enhancement of the calcium K-line in the A-type stars could be the existence of systematic depth-dependent velocity fields in these extended atmospheres. In the case of HD 33579 [A2Ia-O] in the LMC, I have found the calcium $\mathrm{H}$ - and $\mathrm{K}$-line to be asymmetric, thus providing direct observational evidence for the existence of some systematic velocity-field.

(2) I carried out a model atmosphere analysis of HD 7583 in the SMC, according to a similar technique that Groth (1961) applied to analyse $\alpha$ Cyg. As a result the metal abundance came out to be more likely solar within a factor of two, than considerably underabundant. But non-LTE effects, deviations from plane-parallelism, and systematic photospheric velocity fields, not considered in the analysis, are important in these extended atmospheres. These effects may influence the abundance determination and could be a physical 'explanation' for the supersonic 'microturbulence' determined in the analysis as wcll. 